\title{
Neural Network Reconstruction of Single-Photon Emission Computed Tomography Images
}

\author{
John P. Kerr and Eric B. Bartlett
}

\begin{abstract}
An artificial neural network (ANN) trained on highquality medical tomograms or phantom images may be able to learn the planar data-to-tomographic image relationship with very high precision. As a result, a properly trained ANN can produce comparably accurate image reconstruction without the high computational cost inherent in some traditional reconstruction techniques. We have previously shown that a standard backpropagation neural network can be trained to reconstruct sections of single photon emission computed tomography (SPECT) images based on the planar image projections as inputs. In this study, we present a method of deriving activation functions for a backpropagation ANN that make it readily trainable for full SPECT image reconstruction. The activation functions used for this work are based on the estimated probability density functions (PDFs) of the ANN training set data. The statistically tailored ANN and the standard sigmoidal backpropagation ANN methods are compared both in terms of their trainability and generalization ability. The results presented show that a statistically tailored ANN can reconstruct novel tomographic images of a quality comparable with that of the images used to train the network. Ultimately, an adequately trained ANN should be able to properly compensate for physical photon transport effects, background noise, and artifacts while reconstructing the tomographic image.

Copyright $\odot 1995$ by W.B. Saunders Company
\end{abstract}

KEY WORDS: artificial neural network (ANN), backpropagation, probability density function (PDF), singlephoton emission computed tomography (SPECT), statistically tailored neural network.

$\mathbf{F}$ ILTERED BACKPROJECTION algorithms encompass a wide range of analytic and algebraic reconstruction techniques, ${ }^{1-4}$ and are predominantly used in clinical single-photon emission computed tomography (SPECT) image reconstruction..$^{5,6}$ These techniques are used in clinical environments primarily because of their ability to handle incomplete and noisy data and to generate reconstructions in a relatively short period of time. ${ }^{2,7}$ Despite

From The Adaptive Computing Laboratory, Biomedical Engineering Program, Iowa State University, Ames, $L A$.

Address reprint requests to Eric B. Bartlett, PhD, Iowa State University, Adaptive Computing Laboratory, 107 Nuclear Engineering Lab, Ames, IA 50011-2241.

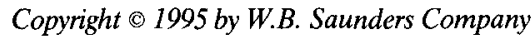

0897-1889/95/0803-0005\$3.00/0 their wide acceptance, the quality of filtered backprojection reconstructions is often degraded by artifacts, attenuation, and scatter effects. ${ }^{8-11}$ Correction for attenuation and scatter effects in the reconstructed images is often used. However, iterative methods as well as compensatory techniques used in conjunction with filtered backprojection typically have high computational costs. ${ }^{12,13}$ In this paper, we show that an artificial neural network (ANN) trained on filtered backprojection reconstructions can interpolate between images in the training set and accurately reconstruct nontraining set images, referred to as novel images, that are equivalent to those produced by the filtered backprojection algorithm. In addition, we introduce a method of deriving statistically tailored activation functions that, used in combination with a backpropagation ANN, can produce high-speed SPECT image reconstructions with statistical error content comparable with that found in the images used to train the neural network. Based on the work presented here, we ultimately hope to show that an ANN trained on highly accurate, iteratively reconstructed images or on phantom images can learn the planar data-to-tomographic image relationship and can subsequently produce novel reconstructions with an accuracy comparable with that achieved with high-cost computational methods, but in a fraction of the time required with those methods.

The backpropagation ANNs that we will use here are often referred to as mapping neural networks because of their ability to learn difficult functions or functional relationships that are otherwise hard to evaluate. ${ }^{14}$ ANNs have been shown to be useful in SPECT and x-ray image diagnostics, ${ }^{15,16}$ image segmentation, ${ }^{17}$ electrical impedance tomography, ${ }^{18}$ and a variety of other medical applications. ${ }^{19-22}$ The advantages of SPECT image reconstruction with an ANN are two-fold: first, a trained ANN can generate a reconstructed image from the planar projections in a few seconds, even on simple serial computers; second, the novel image recon- 
structions will be of a quality comparable to the quality of the ANN training set images.

Once the ANN is trained for image reconstruction, novel planar data need only be fed forward through the network to quickly generate the reconstructed image at the outputs of the ANN. However, backpropagation training of a neural network is a rather inefficient descending-hill search algorithm. ${ }^{23,24}$ The inefficiency in training an ANN stems from the thousands or often several thousands of iterations that the training set data must be presented to the network before it sufficiently learns the input-output relationship. Consequently, while a trained ANN can perform image reconstructions quickly and simply, the one-time training process itself can be very time consuming and may be impractical when very large network architectures are used, or problems requiring large training sets are attempted.

Fortunately, we have taken advantage of the parallel nature of the neural network by implementing the ANN on a parallel computer. The MasPar MP-2 is a single-instruction multipledata massively parallel system that is composed of a $64 \times 64$ interconnected mesh of processing elements (PEs). The functions of nodes in each layer of an ANN distributed on the MP-2 can be executed simultaneously by dedicating a PE to execute the processes of one node in each layer of the network. This greatly improves the time in which an iteration of the ANN training set, or training epoch, can be performed.

In previous work, we and others have shown that image reconstruction with a backpropagation ANN is feasible, ${ }^{25,26}$ and that better generalization and faster training could be achieved with a uniformly distributed, three-phase activation function versus a standard sigmoidal activation function. ${ }^{27}$ In the latter work, we speculated that the optimal activation function for ANN image reconstruction might be related to the probability density function (PDF) of the ANN training data. We propose and empirically test this hypothesis by comparing the ability of identical ANN architectures to generate $64-x$ 64-pixel reconstructions of SPECT images using standard sigmoidal activation functions, and statistically tailored activation functions. We contend that the statistically tailored activation functions, in conjunction with the backpropaga- tion training algorithm, will prove to be the superior transfer functions for the network nodes both in terms of the ANN's ability to learn the reconstruction function from the training set images and to generalize its knowledge for novel image reconstruction.

\section{BACKGROUND: \\ BACKPROPAGATION NEURAL NETWORK}

\section{Fonvard Propagation}

The three-layer, fully interconnected ANN architecture used for image reconstruction in this work consists of 4,096 input nodes to handle the sixty-four 64-quadrant planar data, 4,096 output nodes to produce each $64 \times 64$ slice reconstruction, and a user-selectable number of hidden-layer nodes, as seen in Fig 1. The input layer performs no operation on the data but rather distributes the data via interconnections to all nodes in the middle, or hidden, layer. Each value sent across an interconnection is weighted. The weights associated with the interconnections are the parameters adjusted during training and consequently, represent the knowledge possessed by the ANN. Each node in the hidden layer and output layer sum the values received from interconnections and then on the

\section{Outputs - 64 x 64 SPECT Image}

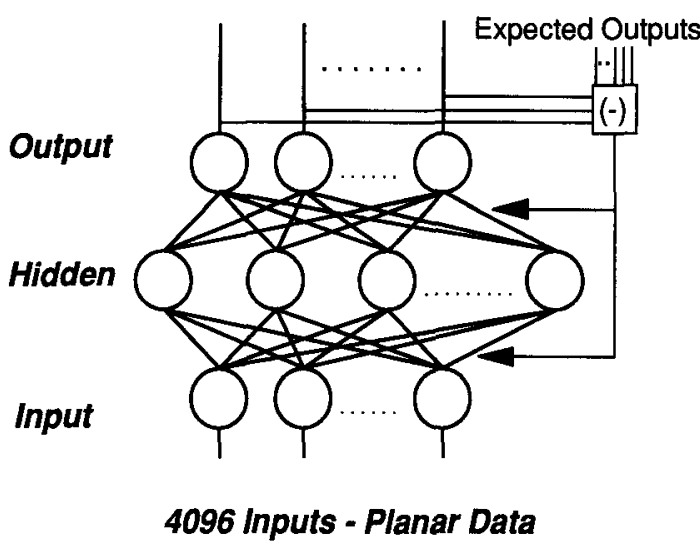

Fig 1. During each training iteration of the 4,096 planar data inputs to 4,096 tomogram outputs, the difference between the expected output and the actual output of the ANN is backpropagated through the network. The weights of the two layers of interconnections are then adjusted according to the delta rule and generalized delta rule to reduce the network error. After repeated training iterations, the ANN will begin to learn the functional relationship between the input vectors and output vectors. 
basis of that value generate an output via an activation function, $\mathrm{f}(\alpha)$.

A sigmoidal activation function similar to the one shown in Fig 2 and defined in equation 1 is typically used with a backpropagation ANN. ${ }^{28}$

$$
f\left(\alpha_{i+1, k}\right)=\beta\left[(1 / \pi) \arctan \left(\alpha_{i+1, k}+\theta\right)+1 / 2\right],
$$

where $\alpha_{i+1, k}=g\left(\Sigma_{j} x_{i, j} w_{i, j}\right)$ for the $k$ th node in layer $i+1 ; x_{i, j}$ is the output of the $j$ th node in the previous layer $i ; w_{i, j}$ is the weight associated with the interconnection from the $j$ th node in the previous layer $i$; $g$ is the gain adjustment of the summed input value; $\beta$ is the gain value of the function; and $\theta$ is the output bias that shifts the zero point of the function.

The ANN training set from which the ANN learns the input-output function must adequately represent the mapping domain of the functional relation..$^{29}$ This means that the training set data must closely approximate the PDF of the output space. The training data are normalized into a 0.1 to 0.9 range because the activation functions are asymptotically limited between 0 and 1 . Normalization of the data allows the network to train to values at the extremes of the output range without having to drive the network outputs into saturation.

\section{Error Backpropagation}

The ANN training set is made up of $\left(\mathrm{X}_{\mathrm{i}}, \mathrm{Y}_{\mathrm{i}}\right)$ data pairs in which $Y_{i}$ is the known functional output associated with the input vector $X_{i}$. In the course of a training iteration, the output produced by an input vector that has been

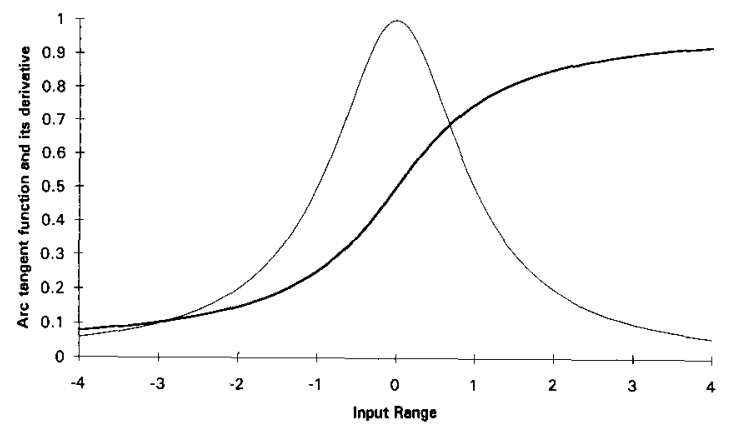

Fig 2. Sigmoidal activation function (-) and its derivative $(-)$ used with standard backpropagation ANNs. Activation functions are used by the hidden-layer and output-layer nodes. The ANN training set data is typically normalized between 0.1 and 0.9 so that outputs near 0 and 1 can be attained by the network without having to drive the output nodes into saturation. forward-propagated through the network is subtracted from the expected output vector. This output difference is backpropagated through the network to make interconnection weight adjustments, thus minimizing the network error. ${ }^{29}$

For this study, the measured error in the networks capacity to accurately recall training set data is calculated as the root-mean-square (RMS) error, C:

$$
\mathrm{C}=\left\{[1 / \mathrm{N} \cdot \mathrm{J}] \cdot\left[\Sigma \Sigma\left(\mathrm{D}_{\mathrm{n}, \mathrm{j}}-\mathrm{Y}_{\mathrm{n}, \mathrm{j}}\right)^{2}\right]\right\}^{1 / 2}
$$

where $J$ is the number of nodes in the output layer; $N$ is the number of patterns in the training set; $D_{n, j}$ is the expected $j$ th output-layer node value for the $n$th training set pattern; and $Y_{n, j}$ is the actual $j$ th output-layer node value for the $n$th training set pattern.

The output vector in this study, is the reconstructed image associated with the planar view inputs. The error in the reconstruction is calculated for each node in the output layer by subtracting the actual output from the expected output. The backpropagation training algorithm adjusts the interconnection weight values according to the delta rule. ${ }^{23,25,19}$

$$
\begin{aligned}
\mathrm{w}_{\mathrm{i}, \mathrm{j} \text { new }}=\mathrm{w}_{\mathrm{i}, \mathrm{j} \text { old }}+ & \eta \sigma_{\mathrm{i}} \mathrm{x}_{\mathrm{i}} \\
& +\alpha\left(\mathrm{w}_{\mathrm{i}, \mathrm{j} \text { old }}-\mathrm{w}_{\mathrm{i}, \mathrm{j} \text { previous }}\right),
\end{aligned}
$$

where $w_{i, j o l d}$ is the present weight value of the interconnection from the $j$ th hidden node to the $i$ th output node and $w_{i, \text { jprevious }}$ is the interconnection weight value before being adjusted to $w_{i, j o l d}$.

To adjust the weight values of the interconnections between the hidden layer and the output layer, we define $\sigma_{i}$ in equation 3 as the error difference in the $i$ th node, $\left(\mathrm{d}_{\mathrm{i}}-\mathrm{y}_{\mathrm{i}}\right)$, multiplied by the associated value of the activation function derivative.

$$
\sigma_{i}=f^{\prime}\left(y_{i}\right)\left(d_{i}-y_{i}\right)
$$

where, for the sigmoidal activation function shown in equation 1 ,

$$
\mathrm{f}^{\prime}\left(\mathrm{y}_{\mathrm{i}}\right)=\mathrm{d}\left[\arctan \left(\mathrm{y}_{\mathrm{i}}\right)\right] / d \mathrm{~d}_{\mathrm{i}}=1 /\left(1+\mathrm{y}_{\mathrm{i}}^{2}\right) \text {. }
$$

Old interconnection weights are adjusted so as to minimize the error between the expected output $d_{i}$ and the actual output $y_{i}$ in equation 3 . The error value is multiplied by a learning rate, 
$\eta$, that governs the magnitude of each adjustment and by the last input across that interconnection, $x_{i}$, that factors the weight adjustment values according to the magnitude of the last input vector. The momentum term $\alpha$ provides an impetus to the weight adjustment to take into account the direction in which the weight was adjusted in the previous training iteration. The momentum term helps the training network to avoid small local minima. ${ }^{24,28}$

The derivative of the arc tangent sigmoidal activation function, $f^{\prime}\left(y_{i}\right)$, shown in Fig 2 , is also used by the network during training to add stability to the network and to prevent too much emphasis in error adjustments being placed on large weight values. ${ }^{23,24}$ The bell-shape of the derivative creates a high degree of activity in nodes whose outputs are in the center of the output range. This shows why the backpropagation ANN is adept as a classifier. As all the outputs approach 0 or 1 , the activity in weight adjustments, as factored by $f^{\prime}\left(y_{i}\right)$, is reduced and the stability of the network training is increased.

The weight values associated with the interconnections from the input layer to the hidden layer do not have the advantage of knowing the expected outputs of the hidden-layer nodes. Consequently, these weight values must be adjusted according to a different value of $\sigma_{i}$ in the generalized delta rule. ${ }^{23,24}$

$$
\sigma_{i}=f^{\prime}\left(x_{i}\right)\left\{\sum_{k=0}^{K}\left[\left(d_{k}-y_{k}\right) w_{i, k}\right]\right\}
$$

where $w_{i, k}$ is the interconnection weight from the $i$ th hidden node to the $k$ th output node.

Instead of using the difference between the expected and actual value of the associated output node, the error associated with a hidden node is the error in the entire output vector weighted with respect to the interconnection weights from that particular hidden node $\left(\Sigma\left[d_{k}-y_{k}\right]\right)$. Again, the input- to hidden-layer interconnection weight adjustments are a function of the derivative of the hidden-layer node activation function, $f^{\prime}\left(x_{i}\right)$.

\section{MATHEMATICAL METHODS}

The backpropagation ANNs used here consist of three layers of nodes. The importance of a three-layer network versus a one- or two-layer network is that we know an ANN solution exists even if the function being mapped is discontinuous. ${ }^{29}$ When an ANN trains to a minima there is no way of knowing whether the network has reached a local minimum, a global minimum, or a large, low-gradient plateau. ${ }^{24}$ Often, if the minimum error to which the ANN is trained is not acceptable for the intended application, a new set of initial weight values is selected and training is restarted.

As mentioned in the previous section, the nodes in a standard backpropagation ANN use a sigmoidal activation function. We also noted that the backpropagation neural network is very adept as a classifier when using a sigmoidal activation function and its derivative. During training, the bell-shaped derivative, as used in the delta rule and generalized delta rule in equations 4 and 6 , produces large changes in the interconnection weights associated with outputs around 0.5 . As the output of a node approaches either 0 or 1 , the changes in the interconnection weights to that node become smaller, and the network becomes more stable. Unfortunately, when training outputs that are continuous in the normalized 0.1 to 0.9 output range, the network tends to become unstable and oscillate about expected output values near 0.5. However, because we assume the training set data is a good representation of the mapping to be learned, the PDF of the data should provide good estimates of the input range PDF and the output domain PDF, and consequently, be utilized to improve ANN training over a continuous range of outputs.

The key characteristic of a good output layer activation function is one that will allow easy training across the continuous range of values and increase the stability to the network as it learns. We contend that an activation function equal to the integral of the inverse estimated PDF for each output node, as in equation 7 , should provide a better training response by the network.

$$
\mathrm{y}_{\mathrm{i}}=\int \mathrm{z}\left(\mathrm{x}_{\mathrm{i}}\right) \mathrm{dx} \mathrm{x}_{\mathrm{i}}
$$

where $z\left(x_{i}\right)$ is the estimated inverse PDF for the $i$ th output node based on the distribution of the nodes training set data values.

For the demonstration of SPECT image reconstruction in this report, a common activation function is used for all output nodes:

$$
\begin{aligned}
f(\alpha)= & \int\left\{1.2 /\left[1+36(\alpha+2.93706)^{2}\right]\right. \\
& +0.4 /\left[1+169(\alpha+2.25918)^{2}\right] \\
& \left.+1.5 /\left[1+1.21(\alpha+1.43482)^{2}\right]\right\} \mathrm{dx} \\
= & \{1.2[(1 / \pi) \arctan [6(\alpha+2.93706)]+0.5] \\
& +0.4[(1 / \pi) \arctan [13(\alpha+2.25918)]+0.5] \\
& +1.5[(1 / \pi) \arctan [1.1(\alpha+1.43482)] \\
& +0.5]\} / 3.1 .
\end{aligned}
$$

Ideally, each output node activation function is derived from the estimated PDF obtained from the training set data for each output node. The single activation function used here is based on the estimated PDF of all pixel values of the tomograms in the ANN training set (see Fig 3). We determined that this was an acceptable approximation of the output layer activation functions because the intent of 


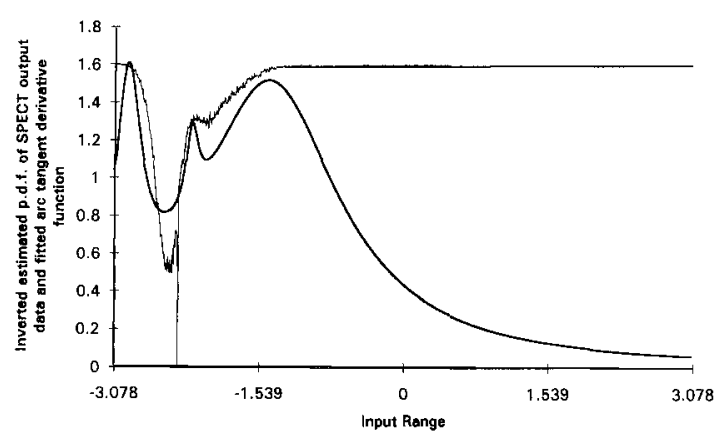

Fig 3. Three arc tangent derivatives were used to fit the inverse statistical distribution of the ANN training set output data. The planar input data distribution was normalized between -3.078 and 3.078 so as to correspond to the 0.1 to 0.9 range of the standard sigmoidal activation function. This was done so that an accurate comparison between the statistically tailored and standard sigmoidal activation functions could be made. SPECT data (-), derivative (-).

the application in this report is to show the attainable improvements in ANN performance with a statistically tailored activation function versus a standard sigmoidal activation function. Also, we will save considerable time in not having to derive activation functions for all 4,096 output nodes if the performance of a common activation function is good enough for the application at hand.

A graph of equation 8 and the three arc tangent functions that comprise the output layer activation function are shown in Fig 4. This activation function shows that by integrating the inverse PDF, an asymptotically increasing function with a greater range of inputs to the most expected outputs is generated. This means that the most frequently occurring output values are far more accessible in the activation function than the more rarely occurring values. As a result, during training, the ANN can better distinguish between the most frequently encountered output values. The theory behind this selection of output-layer activation functions is also supported by the effect on training of the activation function derivative, or the inverse PDF, in the delta rule. As the network trains, the majority of values converge to the expected value range of the activation function, driven by large values of the derivative function. As the common

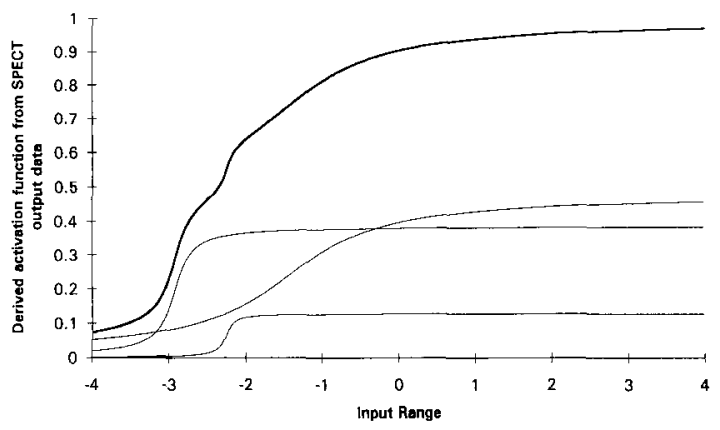

Fig 4. The statistically tailored output-layer activation function (-) is shown as the sum of the three arc tangents $(-)$ obtained by integrating the inverse output data distribution functions. values reach the expected output range, the activity of the derivative functions in the adjustment of weight values decreases and the network stabilizes. The reduced activity in weight adjustments also allows the network, during additional training, to distinguish between output values without experiencing large oscillations, as often seen with a standard sigmoidal function and derivative.

On the other hand, an activation function derived from integrating the estimated PDF of the training set input vectors is best suited for the hidden layer.

$$
\begin{aligned}
f(\alpha)= & \int\left\{1 /\left[1+196(\alpha+3.077)^{2}\right]\right. \\
& +0.38 /\left[1+33.64(\alpha+2.587)^{2}\right] \\
& \left.+0.58 /\left[1+2.89(\alpha+1.760)^{2}\right]\right\} \mathrm{dx} \\
= & \{0.55[(1 / \pi) \arctan [14(\alpha+3.077)]+0.5] \\
& +0.38[(1 / \pi) \arctan [5.8(\alpha+2.620)]+0.5] \\
& +(1 / \pi) \arctan [1.7(\alpha+1.760)]+0.5\} / 1.96 .
\end{aligned}
$$

Each node of the hidden layer receives a weighted sum of all elements in the input vector. Accordingly, the input vector whose sum falls in the mostly densely distributed part of the PDF, as shown in Fig 5, will occupy a larger portion of the output range than a less frequently encountered vector sum, (see Fig 6). By delivering a broad range of values from the dense areas of the input sum PDF to the output layer, the output nodes have a better capacity to distinguish between closely distributed outputs, and thus, should provide a better ANN model for training and generalizing.

Each of the statistically tailored activation functions are composed of the sum of three sigmoidal functions. The combination of three functions proved sufficient to approximate the cumulative distribution functions (CDFs) of the SPECT training set data used in this study. Exact fits to the training set CDFs are not necessary because they themselves are approximations of the actual CDFs. We also expect that any discrepancies in activation function approximations will be compensated for through ANN training. We have used sigmoidal functions to retain the training advantages of using the S-shaped sigmoidal, nonlinear elements, which have been shown to provide superior generalization

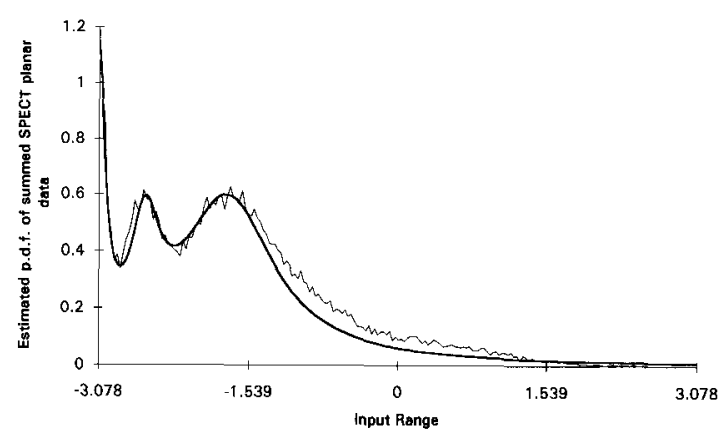

Fig 5. Three arc tangent derivatives were used to fit the statistical distribution of the ANN training set input data. The planar input data distribution was normalized between -3.078 and $\mathbf{3 . 0 7 8}$ so as to correspond to the 0.1 to 0.9 range of the standard sigmoidal activation function. Planar data $(-)$, derivative (-). 


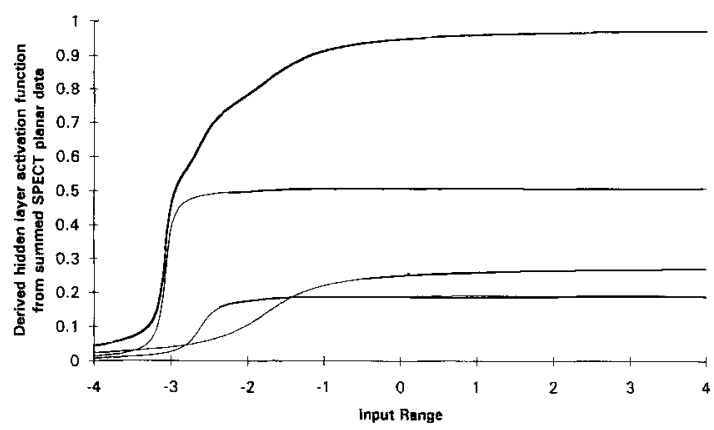

Fig 6. The statistically tailored hidden-layer activation function (-) is shown as the sum of the three arc tangents (-) obtained by integrating the input data distribution functions.

capabilities to linear and polynomial functions. ${ }^{23,29}$ In addition, we maintain a link to organic cerebral neurons that show a natural sigmoidal relationship between the input wave density to a neuron and the output pulse density of a neuron. ${ }^{30}$

We have previously described how a standard backpropagation ANN with a sigmoidal activation function is adept as a classifier network. This example also provides a good argument for using the statistically-based activation functions we have proposed here. As can be seen from Fig 2, the sigmoidal function derivative is essentially the inverse distribution of the expected outputs for a classifier network because only values of 1 and 0 are typically desired from the network. For the hidden-layer nodes, the sigmoidal derivative approximates a Gaussian distribution. Because each node in the hidden layer receives a weighted sum of all the inputs, this is probably a good estimate of the input data distribution, particularly for problems where the inputs, like the classifier output, are binary. Consequently, the sigmoidal activation function and derivative are well suited for training problems for which the expected output node values are binary. Because each output node in the SPECT reconstruction ANN must be readily trainable across a continuous range of outputs, the standard sigmoidal ANNs are not expected to perform as well as the ANNs with statistically tailored activation functions.

\section{RESULTS}

\section{SPECT Training Set Data}

Cardiovascular SPECT images were used to train the neural networks and to test the trained ANN's ability to reconstruct novel $64-\times 64$ pixel images. The planar view images were each two-dimensionally filtered using a Butterworth filter with a critical frequency of 0.5 cycles $/ \mathrm{cm}$ and a power factor of 15 . The transverse image slices were then reconstructed using conventional filtered backprojection.

The ANN training set consisted of every other slice of a 16-slice cardiac SPECT image, and the 64 planar views of each image slice. The
SPECT images not used in the training set were used to determine each trained ANN's ability to accurately reconstruct novel tomograms from their associated planar data. The novel reconstructions can then be compared with those attained from the Butterworth filtered backprojection algorithm, to determine if the quality of the ANN reconstructions is directly associated to the quality of the training set images. In addition, a second series of fifteen cardiac SPECT images were used to test the extent of the ANN's ability to reconstruct images uncorrelated to the small eight-image training set.

\section{Activation Function Evaluation}

Four architectures with randomly selected hidden-layer sizes were constructed to evaluate the training and generalization capabilities of the statistically tailored activation function ANNs versus the standard sigmoidal activation function ANNs. Each architecture, using the two activation function types, were trained for 2,000 iterations on the eight SPECT image training set. The minimum root mean square (RMS) errors attained by each network are listed in Table 1 . In each case the statistically tailored ANNs trained to a lower RMS error than their counterpart standard sigmoidal ANNs. An RMS error versus training epoch comparison of the statistically tailored ANN and the standard sigmoidal ANN with 4,096 $\times$ $484 \times 4,096$ architectures is shown in Fig 7. As was the case with all architectures, the standard sigmoidal ANN initially trains quickly and then plateaus, whereas the statistically tailored ANN

Table 1. Minimum RMS Errors

\begin{tabular}{ccc}
\hline ANN Architecture & $\begin{array}{c}\text { Activation } \\
\text { Function Type }\end{array}$ & $\begin{array}{c}\text { RMS Error on } \\
\text { Training Set Data }\end{array}$ \\
\hline $4,096 \times 324 \times 4,96$ & Statistically tailored & 0.010529 \\
& Standard sigmoidal & 0.020116 \\
$4,096 \times 484 \times 4,096$ & Statistically tailored & 0.008703 \\
& Standard sigmoidal & 0.012740 \\
$4,096 \times 625 \times 4,096$ & Statistically tailored & 0.009309 \\
& Standard sigmoidal & 0.018772 \\
$4,096 \times 961 \times 4,096$ & Statistically tailored & 0.011972 \\
& Standard sigmoidal & 0.020333 \\
\hline
\end{tabular}

RMS errors were attained in 2,000 training epochs for the four architectures and the two activation function types. For each architecture, the neural network using statistically tailored activation functions trained to a lower RMS error than any of the ANNs using the standard sigmoidal activation function. The hidden-layer size for each architecture was arbitrarily chosen. 


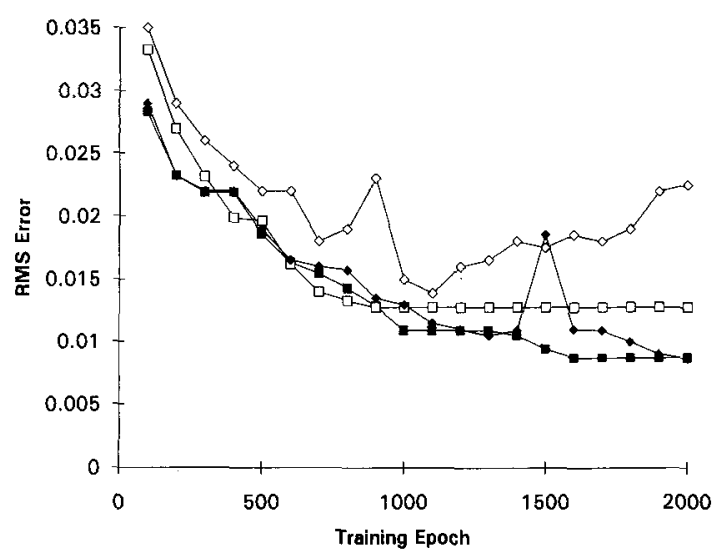

Fig 7. The RMS error in recalling the training set reconstructions and the novel eight-image set for the 4,096-node $x$ 484-node $\times$ 4,096-node architecture is shown with respect to training epoch for both the statistically tailored ANN and the standard sigmoidal ANN. As was the case with the other three architectures trained, the statistically tailored ANN reached a lower RMS error for both the training set images (StatANN, a) and the novel eight-image recall set (StatGen, $\$$ ) than did the standard sigmoidal ANN (SigmANN, $\square$, and SigmGen, $\diamond$ ). The statistically tailored ANN also continued to reduce its RMS error throughout the 2,000 training iterations. The standard sigmoidal ANN reached its minimum RMS error after approximately 1,000 iterations and did not improve over the last 1,000 training epochs.

trains slightly slower in the first few hundred iterations, but then continues past the standard sigmoidal plateau, where it eventually plateaus at an RMS error of approximately 0.009 .

A more critical measure of the ANN's reconstruction capabilities is in the trained ANN's ability to accurately reconstruct novel images. The best RMS errors achieved by each trained ANN on the eight SPECT images not used to train the networks are listed in Table 2. Here

Table 2. Minimum Average RMS Error

\begin{tabular}{ccc}
\hline ANN Architecture & $\begin{array}{c}\text { Activation } \\
\text { Function Type }\end{array}$ & $\begin{array}{c}\text { RMS Error on } \\
\text { Novel lmage Set }\end{array}$ \\
\hline $4,096 \times 324 \times 4,096$ & Statistically tailored & 0.011389 \\
& Standard sigmoidal & 0.020454 \\
$4,096 \times 484 \times 4,096$ & Statistically tailored & 0.008619 \\
& Standard sigmoidal & 0.013911 \\
$4,096 \times 625 \times 4,094$ & Statistically tailored & 0.010022 \\
& Standard sigmoidal & 0.016678 \\
$4,096 \times 961 \times 4,096$ & Statistically tailored & 0.014676 \\
& Standard sigmoidal & 0.023611 \\
\hline
\end{tabular}

RMS errors were attained by each ANN architecture reconstructing the eight novel images. Each statistically tailored ANN was able to reconstruct the eight first-patient tomograms with less error than the same architecture standard sigmoidal ANN. Upon visual inspection, only the statistically tailored ANNs with 484 and 625 hidden nodes produced images that accurately represented the features of the novel images. again the statistically tailored ANNs significantly outperformed the standard sigmoidal ANNs. For the 4,096 $\times 484 \times 4,096$ architecture, the statistically tailored ANN reconstructed the novel images with less than 0.0094 error from the expected Butterworth-filtered backprojection reconstructions. No standard sigmoidal ANN could reconstruct the novel images with less than 0.014 error. A comparison of the generalization ability of a statistically tailored ANN and standard sigmoidal ANN during training is shown in Fig 7. In both instances, the generalization ability of the networks initially follows the RMS error of the training set. However, after reaching the minimum RMS error, the generalization ability of the standard sigmoidal network decreases with additional training because the network begins to memorize the training set and, thus, recalls training set reconstructions when presented with novel planar data inputs. However, for the statistically tailored ANN the generalization ability of the ANN continues to improve, even after it appears to begin memorizing.

A novel image reconstruction by both a statistically tailored ANN and a standard sigmoidal ANN are shown, along with filtered backprojection reconstructions, in Fig 8. This visual comparison of the ANN reconstructions clearly shows the greater accuracy of the statistically tailored ANN compared with that of the standard sigmoidal ANN or the filtered backprojection reconstruction without Butterworth filtering.

The second patient planar data set was reconstructed by the best of each type of ANN to determine the extent of their reconstruction abilities, given the limited single-patient scope of the training set. The 15 -tomogram set was reconstructed with 0.035686 RMS error by the statistically tailored ANN and to 0.072034 RMS error by the standard sigmoidal ANN. For each type of network, the 484 hidden-node architecture was used.

\section{DISCUSSION}

The results of Tables 1 and 2 show that the statistically tailored neural network clearly outperforms the standard sigmoidal ANN. Although the RMS error differences between the two network types appear small, these numbers 
Fig 8. Each of the reconstructions are based on the same planar data. A was reconstructed the same as all the images in the ANN training set, via filtered backprojection with two-dimensional (2D) Butterworth prefiltering. $B$ is the statistically tailored 4,096-node $X$ 484-node $\times$ 4,096-node ANN reconstruction, with 0.008712 RMS error from the expected image, $A$. $C$ is the standard sigmoidal 4,096-node $\times$ 484-node $\times$ 4,096-node ANN reconstruction, with 0.014341 RMS error from the expected image, $A$. $C$ was produced via filtered backprojection without the $2 \mathrm{D}$ Butterworth prefiltering. The statistically tailored ANN reconstruction closely matches the expected image, $A$.

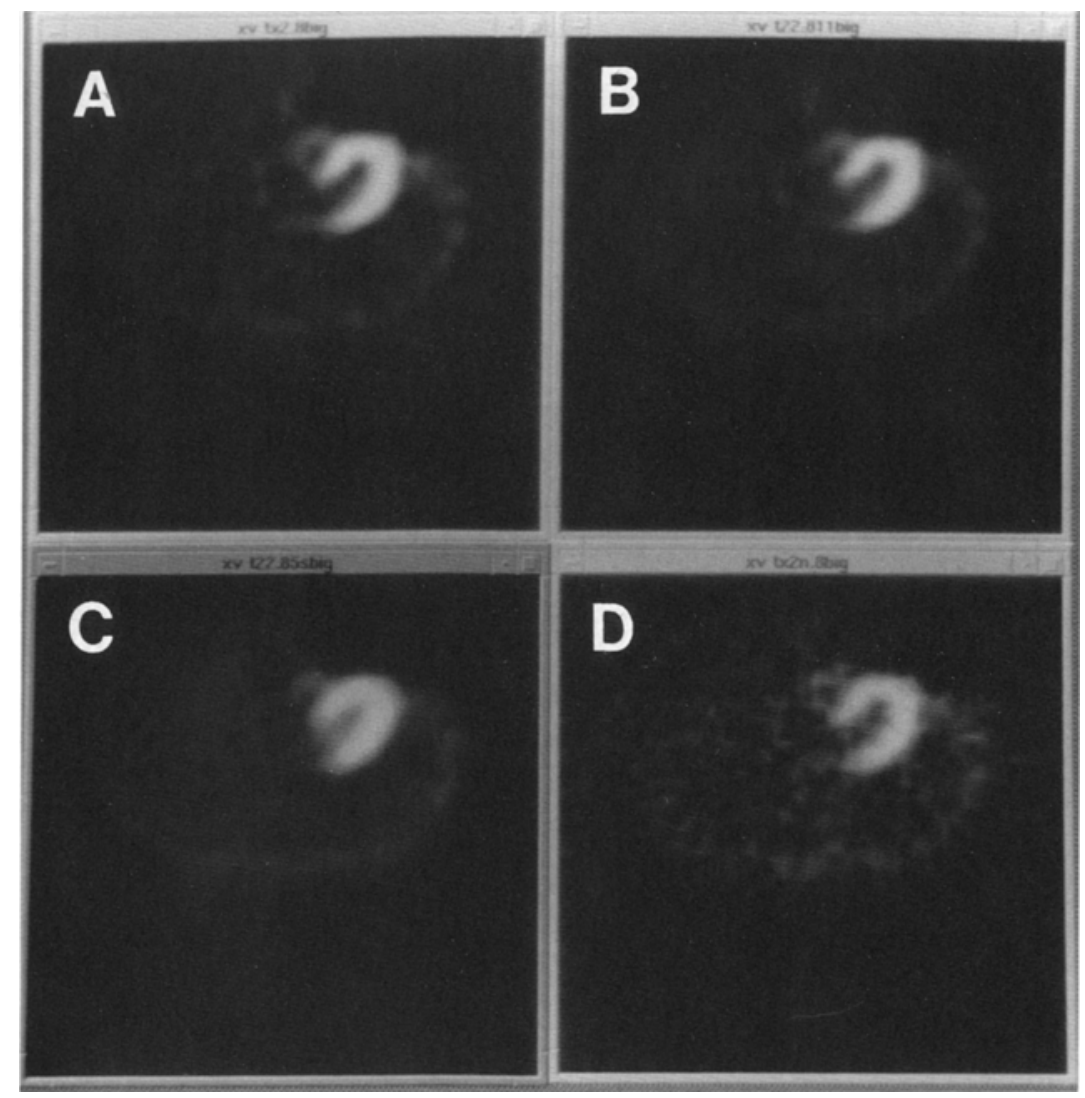

can be deceptive. The difference between the images reconstructed with 0.008619 error and the images reconstructed with 0.013911 error is significant. As seen in Fig 8, the statistically tailored ANN novel image reconstruction is a very accurate representation of the expected image, and not merely a reproduction of a training set image. On the other hand, the standard sigmoidal ANN reconstruction of the same novel image is far less representative of the expected image, and possesses many of the features of the closed training set images. Even though the two reconstructions of the same novel image differ by less than $0.01 \mathrm{RMS}$, that 0.01 is significant because the cardiac portion of the $64 \times 64$ image is small. As a result, the statistically tailored ANN is able to generalize its knowledge attained during training to more accurately produce high-quality reconstructions.

The statistically tailored neural networks proved to be a superior backpropagation ANN method for SPECT image reconstruction when compared with the standard sigmoidal back- propagation ANNs. Each statistically tailored ANN trained to a significantly lower RMS error than the same architecture standard sigmoidal ANN. In addition, all four statistically tailored ANNs achieved RMS errors lower than the best standard sigmoidal ANN. Furthermore, the standard sigmoidal ANNs tended to reach a plateau at the minimum RMS error in approximately 1,000 training epochs, whereas the statistically tailored ANNs, which also reach a plateau at approximately 1,000 training iterations continued to improve slowly over the next 1,000 iterations, as seen in Fig 7.

Likewise, the statistically tailored ANNs showed excellent generalization capabilities. Again, the statistically tailored ANNs reconstructed the novel image set to much lower RMS errors than the standard sigmoidal ANNs with the same architecture. One surprising, and potentially invaluable, characteristic of the statistically tailored ANNs is seen in Fig 7. The figure shows that the standard sigmoidal ANN reaches a minimum generalization RMS error in 1,100 training epochs, and then gradually gets 
worse with additional training. This is a common and usually expected characteristic of the backpropagation ANN. ${ }^{28}$ However, the statistically tailored ANN, after appearing to lose its generalization ability at 1,500 training epochs as shown in Fig 7, quickly recovers, and by the two-thousandth training epoch, the novel images set RMS error and the training set RMS error are practically identical. Perhaps the loss in generalization was a consequence of the training set RMS error dropping to a lower plateau at the 1,500 training epoch mark. Note however, that for the two training set RMS error plateaus in the 1,000 to 2,000 training epoch range, the generalization RMS error improves with additional training. If this is indeed a characteristic of the statistically tailored neural network, then overtraining of a network is much less likely to occur. Thus, the generalization RMS error need not be as closely monitored during training.

Of the four ANN architectures trained, one network was able to reconstruct the novel image set with less than 0.01 RMS error, and one network reconstructed the novel image set right at 0.01 RMS error, As was shown by Fig 8, a reconstruction with 0.008712 RMS error is an accurate and valuable image, but with 0.014341 RMS error, the same image may not accurately represent the expected image. Even with the statistically tailored ANN there is no assurance that the selected hidden-layer size for an ANN is trainable to an RMS error below 0.01. Undoubtedly, the optimal ANN architecture for reconstructing every slice of a completely novel SPECT scan will differ from the best architecture presented here. However, a dynamic node architecture (DNA) method has been developed $^{31}$ and applied practically ${ }^{32}$ that guarantees finding the optimal architecture for a given training set. Whether a DNA neural network for image reconstruction can be trained in a reasonable amount of time on a parallel computer is yet to be determined.

The reconstruction of the uncorrelated second patient images was not of the same high quality as the first novel image set. This was not unexpected given the limited size and scope of the training set. This shows some of the future aspects of ANN SPECT that need to be addressed before a clinically viable image recon- struction neural network can be produced. However, given the greater error in the second patient reconstructions, the statistically tailored ANN did show a propensity for properly reconstructing the images in the regions of the $64 \times$ 64 tomogram that were trained across a broader range of pixel values. This was particularly true in the region of the tomograms where the first patient heart was located.

\section{CONCLUSIONS}

In the introduction we speculated that a properly trained neural network could produce novel image reconstructions of a quality comparable with that of the images used to train the network. The results we have presented have verified that hypothesis. In Fig 8 , a filtered backprojection reconstruction of the novel image, without the two-dimensional Butterworth filtering of the planar data, was included to reinforce our hypothesis. Because the network was trained on images with prefiltering, the network essential learned to perform the Butterworth prefiltering of the planar data and reconstruct a tomogram on the basis of that data. Likewise, an ANN trained on reconstructions produced via a statistically more accurate method, can produce novel images of comparable quality, and in a fraction of the time.

Two potential limitations of this reconstruction method are in the orientation of the planar data and the ultimate size of the ANN training set. The first limitation involves determining how well the neural network can handle planar data that does not begin a scan at the same relative location to the patient. If this is in fact a problem the ANN cannot handle, the data may be repositioned before ANN reconstruction to alleviate the neural networks problem. The second potential limitation deals with the scope of the ANN's generalized knowledge. For example, can a network trained exclusively on normal cardiac SPECT images accurately reconstruct abnormal images? As shown with the second patient image set, the ANNs will need a much larger training set to achieve all image reconstruction capabilities. But if the training set must have an example of every cardiac anomaly to produce accurate reconstructions, the training set will be impractically large. However, the results presented here show that 
an example of every slice of a full SPECT image is not needed in the training set for the ANN to accurately reconstruct novel slices.

Improvements to the RMS error results could have been obtained in several ways, such as reducing the $64 \times 64$ inputs and outputs to the actual 64-pixel diameter circular gamma camera field of view. However, our goal was to show that a statistically tailored backpropagation neural network could accurately produce novel SPECT image reconstructions, and this was achieved without the aforementioned improvements. In addition, we were able to show that the ANNs can interpolate between images to accurately reconstruct novel images. The next step will be to show that the networks can interpolate between pixel values in the $64 \times 64$ tomograms so that any possible tomogram can be accurately reconstructed.
Future work will strive to overcome the aforementioned potential limitations of ANN image reconstruction, and to determine if a neural network can correct for scatter and attenuation effects in its reconstructions. The nonlinear nature of neural networks may enable them to compensate accurately for nonlinear sources of error, such as attenuation. Ultimately, the speed and trainability of neural networks for image reconstruction may prove them to be valuable tools for clinical applications.

\section{ACKNOWLEDGMENT}

We thank Ames Laboratory's Scalable Computing Laboratory and its director, John Gustafson, for providing access to the MasPar MP-2 and for their many insights into parallel coding; William McLeud, Administrator at Story County Hospital, Iowa, Dr Andrea McGuire, and David Nowak, General Electric Medical Systems, for providing the SPECT image data used in this work.

\section{REFERENCES}

1. Jaszczak R: Tomographic radiopharmaceutical imaging. Proc IEEE 76:1079-1094, 1988

2. Cho Z, Jones J, Singh M: Foundations of Medical Imaging. New York, NY, Wiley, 1993

3. Kak AC, Slaney M: Principles of Computerized Tomographic Imaging. New York, NY, IEEE Press, 1988

4. Andersen A: Algebraic reconstruction in CT from limited views. IEEE Trans Med Imaging 8:50-55, 1989

5. Parker JA: Image Reconstruction in Radiology. Boca Raton, FL, CRC Press, 1990

6. Macovski A: Medical Imaging Systems. Englewood Cliffs, CA, Prentice-Hall, 1983

7. Greer K, Jaszczak R, Coleman R: An overview of a camera-based SPECT system. Med Phys 9:455-463, 1982

8. Chang LT: A method for attenuation correction in radionuclide computed tomography. IEEE Trans Nucl Sci NS-25:638-643, 1978

9. Oppenheim BE: Scatter correction for SPECT. J Nucl Med 25:928-929, 1984

10. Faber TL, Lewis MH, Corbett JR, Stokely EM: Attenuation correction for SPECT: An evaluation of hybrid approaches. IEEE Trans Med Imaging MI-3:101-107, 1984

11. Floyd CE, Jaszczak RJ, Greer KL, Coleman RE: Deconvolution of compton scatter in SPECT. $J$ Nucl Med 26:403-408, 1985

12. Chornoby ES, Chen CJ, Miller MI, et al: An evaluation of maximum likelihood reconstruction for SPECT. IEEE Trans Med Imaging 9:99-110, 1990

13. Beck J, Jaszczak R, Coleman R, et al: Analysis of SPECT including scatter and attenuation using sophisticated Monte Carlo modeling methods. IEEE Trans Nucl Sci NS-29:506-511, 1982

14. Hecht-Nielsen R: Theory of the backpropagation neural network. International Joint Conference on Neural Networks (IJCNN) 1:593-605, 1989

15. Fujita H, Katafuchi T, Uehara T, et al: Application of artificial neural network to computer-aided diagnosis of coronary artery disease in myocardial SPECT bull's-eye images. J Nucl Med 33:272-276, 1992

16. Wu Y, Giger ML, Doi $K$, et al: Artificial neural networks in mammography: Application of decision making in the diagnosis of breast cancer. Radiology 187:81-87, 1993

17. Coppini G, Poli R, Rucci M, et al: A neural network architecture for understanding discrete three-dimensional scenes in medical imaging. Comput Biomed Res 25:569-585, 1992

18. Guardo R, Boulay C, Bertrand M: A neural network approach to image reconstruction in electrical impedance tomography. Proceedings of the International Conference of IEEE-Eng Med Biol Soc XIII:14-15, 1991

19. Wu Y, Doi K, Metz C, et al: Simulation studies of data classification by artificial neural networks: Potential applications in medical imaging and decision making. $\mathbf{J}$ Digit Imaging 6:117-125, 1993

20. Brotherton T, Pollard T, Simpson P, et al: Classifying tissue and structure in echocardiograms. IEEE Eng Med Biol 13:754-760, 1994

21. Hiraiwa A, Shimohara K, Tokunaga Y: EEG topography recognition by neural networks. IEEE Eng Med Biol 9:39-42, 1990

22. Garg S, Floyd C: The training of an artificial neural network for a detection task in medical images. Proceedings of the International Conference IEEE-Eng Med Biol Soc XIII:1407-1408, 1991

23. Widrow B, Lehr MA: 30 years of adaptive neural networks: Perceptron, madaline, and backpropagation. Proc IEEE 78:1415-1441, 1990

24. Haykin S: Neural Networks: A Comprehensive Foundation. New York, NY, Macmillian, 1994

25. Kerr JP, Bartlett EB: SPECT reconstruction using a backpropagation neural network implemented on a mas- 
sively parallel SIMD computer. Proc IEEE-ComputerBased Medical Systems 5:616-621, 1992

26. Floyd CE: An artificial neural network for SPECT image reconstruction. IEEE Trans Med Imaging 10:485487, 1991

27. Kerr JP, Bartlett EB: High-speed reconstruction of SPECT images with a tailored piecewise neural network. IEEE Nucl Sci Symp Med Imaging 2:1317-1321, 1993

28. Lippmann RP: An introduction to computing with neural nets. IEEE Acoustics, Speech and Signal Processing Magazine April 1987, pp 4-22
29. Hecht-Nielsen R: Neurocomputing. Reading, MA, Addison-Wesley, 1990

30. Freeman W: The physiology of perception. Sci Am 264:78-85, 1991

31. Bartlett EB: Dynamic node architecture learning: An information theoretic approach. Neural Networks 7:129140,1994

32. Bartlett EB: Analysis of chaotic population dynamics using artificial neural networks. Chaos Solitons Fractals 1:413-421, 1991 